\title{
Editorial: On IRIE Vol. 22
}

Many science fiction phantasies already claimed that one day machines will be superior to human beings and computers will finally take over. But unlike in Stanley Kubrick's '2001' or Asimow's 'I, Robot' the latest developments in the Internet of Things (IoT) give reason to suggest that if this will happen it won't be necessarily machines that physically resemble human beings with legs, bodies, voices etc. that will do the job (robots in the classical sense). If, then it will be more like in Matrix - the physicality of the necessary intelligence (i.e. computing power) will vanish as it will be incorporated into the physical world of our daily life itself. It won't be separate machine entities that will dominate the human kind but it will be by the embedding of computing power into the ordinary things of our daily life and their being connected with each other to form a virtual pervaded living space. A living space that then could not only be paradise (optimized by the computing power embedded to the best for mankind) or hell (used to encage and enslave its inhabitants) but even more also a pure illusion (encaged and enslaved inhabitants that are made believe and even sense realistically that they are in paradise).

This is what philosophically the Internet of Things is all about: Things won't be physical things anymore that are independent objects for the examination, exploration and manipulation of an equally independent subject. Things will be what is presented to the subject and the subject is what the computed presentation presupposes 'on the other side': a user, a monitored, a ... . Thus, if the things change in the IoT we will change. And thus, the underlying philosophical subject-object paradigm has to change as well taking this interplay into account. Again, not only theoretically (as depicted in science fiction far from any possible reality) but very practically regarding our daily life: how we automate our homes, how we care for elder people, the way we monitor our children, the concepts we use to organize life in (smart) cities etc. For the good (of who), for the bad (according to what norm)? This is the ethical challenge raised by the IoT and this issue presents some very interesting answers to it and where not complete answers yet very helpful outlines for possible answers an 'Ethics for the IoT' $^{\prime}$ can give and must give (rather sooner than later).

Yours,

the editors. 